\title{
Erosive vulvar lichen planus and risk of vulvar neoplasia: retrospective case series review
}

\author{
Joana Lyra ${ }^{1}$, Catarina Melo $^{2}$, Rita Figueiredo ${ }^{3}$, Rita Polónia-Valente ${ }^{3}$, Vera Falcão ${ }^{3}$, Jorge \\ Beires $^{3}$, and Pedro Vieira-Baptista ${ }^{4}$ \\ ${ }^{1}$ Sao Joao Hospital \\ ${ }^{2}$ Centro Hospitalar Universitário de São João \\ ${ }^{3}$ Centro Hospitalar Universitario de Sao Joao \\ ${ }^{4}$ Centro Hospitalar de Sao Joao
}

May 27, 2020

\begin{abstract}
Objective: To assess the risk of vulvar cancer and precursors in a cohort of women with vulvar lichen planus (LP) and the clinical and therapeutic features of these patients. Design: retrospective case series review Setting: Lower genital tract Unit of a tertiary hospital in Porto, Portugal Participants: 127 women with the diagnosis of vulvar LP, followed in one institution during a period of eleven years - January 2008 until December 2018. Main outcome measures: Demographic and clinical data were evaluated, as well as treatment, follow-up and histology results. Results: A total of 127 women were diagnosed with vulvar LP. The mean follow-up time was 3.9?0.5 years (range 1-11 years). Ultra-potent topical corticosteroids were first line treatment in $91.8 \%(\mathrm{n}=112)$, with 32 cases $(25.2 \%)$ needing an alternative treatment. An overall of 30 biopsies were performed, in 19 women $(15 \%)$. Vulvar HSIL was diagnosed in $3(2.4 \%)$ women, $2(1.6 \%)$ of which were later diagnosed with vulvar squamous cell carcinoma. No cases of differentiated vulvar intraepithelial neoplasia were observed. Conclusion: Pre-malignant/malignant transformation in women with vulvar LP under surveillance and compliant with treatment is low. A close follow-up appears to be crucial to prevent future malignancy. Biopsies should be performed whenever a suspicious lesion appears during the follow-up.
\end{abstract}

\section{Introduction}

Vulvar lichen planus (LP) is an inflammatory disorder that affects mucocutaneous tissues, including vulva, vagina and other non-genital regions, such as oral mucosa, skin and esophagus. LP has an estimated prevalence of $0.5-3.7 \%$, with erosive LP being the most prevalent form. The etiology of this condition is still not fully understood, but it is considered to be an autoimmune disease with a T-cell-mediated pathogenesis, with a possible role for some exogenous factors, such as nonsteroidal anti-inflammatories and antihypertensive drugs. ${ }^{1}$

Vulvar squamous cell cancer (VSCC) can arise from two independent premalignant pathways: a) one HPV related (30-50\% of all cases), and having high-grade squamous intraepithelial lesions (HSIL) as the precursor lesion; b) the other, HPV independent, related to vulvar dermatosis (lichen sclerosus), and having differentiated vulvar intraepithelial neoplasia (dVIN) as the precursor lesion. ${ }^{2-6}$ While those two pathways present as concurrent in VSCC carcinogenesis, there are reports of HPV-related cancers in women with LP, probably due to HPV reactivation or predisposition to acquire new HPV infection, which eventually can originate HSIL. ${ }^{4,5}$

There is an association between LP and cancer in the upper gastrointestinal tract neoplasia (oral cavity, tongue, esophagus). ${ }^{7}$ However, the risk of VSCC in women with vulvar LP is still controversial. There are 
studies describing an incidence of VSCC two times higher in women with vulvar $\mathrm{LP}^{7,8}$, while another study focusing on HPV-independent vulvar cancer did not find any evidence of hypertrophic, classic or erosive LP in VSCC specimens. ${ }^{2}$ Importantly, in one study that associated LP to VSCC, the disease tended to be more aggressive when vulvar LP was present (higher rate of regional lymph node metastases at presentation and a rate of local recurrence of $40 \%) .^{8}$

In face of contradictory evidence when revisiting VSCC and LP, our study aimed to analyze a series of women with vulvar LP followed during an 11 years period, to assess the risk of developing preinvasive and invasive vulvar lesions.

\section{Materials and Methods}

A retrospective cohort study including all the women followed in the Lower Genital Tract Disease Unit of Centro Hospitalar Universitário São João during a period of eleven years (January 2008-December 2018), with the diagnosis of vulvar LP was performed. Patients were referred by the primary care providers, emergency room or from other consultations. The diagnosis of LP was made based on clinical features and/or on histology, according to the ISSVD recommendations..$^{9-12}$

Data were collected through consultation of the electronic files of each patient, where all the information is recorded. We retrieved information on the age at diagnosis, smoking habits, menopause status and the use of menopause hormone therapy. Presenting symptoms and anatomical changes observed in physical examination that contributed to the diagnosis of LP were also evaluated. Follow-up was made, at least, annually. Shorter interval visits were performed whenever deemed necessary: either due to poor control of symptoms or presence of suspicious lesions. Data on the treatments performed, both topical and systemic and the need to escalate therapy for uncontrolled disease were also evaluated. We usually advocate a scheme of application of clobetasol propionate (CP) once a day for 4 weeks, followed by once every other day for another 4 weeks, and finally twice a week for another 4 weeks, completing 3 months of initial treatment. Manutention therapy is usually recommended; patients are advised to use up to $60 \mathrm{~g}$ of $\mathrm{CP} /$ year.

Whenever a suspicious lesion was found during follow-up or when first line treatments were not effective in controlling the disease, vulvar biopsies were performed. The results of all biopsies performed were retrieved and analyzed.

Descriptive and inferential analysis were performed using IBM SPSS Statistics Version 21. We performed a descriptive analysis, with continuous variables summarized as means with standard deviations and categorical variables as percentages of patients in each category.

The study was approved by the ethics committee of the institution $\left(\mathrm{n}^{\mathrm{O}} 182 / 19\right)$.

\section{Patient and public involvement}

This was a retrospective case series study and no patients were involved in the study design, setting the research questions, or the outcome measures directly. Also, patients were not asked to advise on interpretation or writing up of results.

\section{Results}

Demographic data

During the evaluated time period, 127 women had a diagnosis of LP. This population represented 8.4\% $(127 / 1515)$ of all patients evaluated at the vulvar diseases' clinic. Mean age was 59.0 22.9 years (17-90 years-old); most women were older than 50 years $(78 \%, \mathrm{n}=99)$ and post-menopausal $(74.8 \%, \mathrm{n}=92)$. Among the latter, $13.3 \%(\mathrm{n}=11)$ were on hormone therapy.

We had information about body mass index in 83 patients and, of those, 37 (45.1\%) were obese (BMI [?] $\left.30 \mathrm{Kg} / \mathrm{m}^{2}\right)$. Smoking was reported by $5(4.1 \%)$ women.

Presenting symptoms and anatomical changes 
The most frequent symptoms were pruritus $(77.8 \%, \mathrm{n}=91)$ and burning $(44.4 \%, \mathrm{n}=52)$. Less common symptoms included: dysuria, perianal itching, and vulvar pain (Table 1). Fourteen women (12.2\%) reported abnormal vaginal discharge, which could be part of vaginal LP involvement. In terms of vulvar changes, the most frequent findings were effacement of the labia minora in $82.5 \%(\mathrm{n}=104)$, followed by erythema $(69.8 \%, \mathrm{n}=88)$ and phimosis $(54 \%, \mathrm{n}=68)$. The other alterations were less frequent and comprised: fissures, hyperkeratosis, erosions, depigmentation, vaginal stenosis, hyperpigmentation, perianal involvement, edema and purple papules (Table 1).

Table 1. Description and frequencies of presenting symptoms and anatomical changes.

\section{Treatments}

The first line treatment was ultra-potent topical corticosteroid (CP) in $91.8 \%$ ( $\mathrm{n}=112)$. In 32 cases $(25.2 \%)$, a second line treatment was necessary to control symptoms and in 8 cases $(6.3 \%)$ more than one change of treatment was needed. As options, we used more often an alternative topical corticosteroid (hydrocortisone, betamethasone and triamcinolone), followed by calcineurin inhibitors in 10 cases (8.2\%), methotrexate in 4 cases $(3.3 \%)$, retinoids in 2 cases $(1.6 \%)$ and systemic steroids in one case $(0.8 \%)$. In one case, a fourchette plasty was performed to treat dyspareunia associated with synechia.

Regarding adverse effects and complications of the treatments used, 18.8\% ( $\mathrm{n}=21)$ developed a vulvovaginal yeast infection (all associated with ultrapotent topical corticosteroids use) and, in one woman, retinoids had to be stopped due to suicidal ideation.

Follow-up, biopsies and results

The mean time of follow-up time was $3.9 \pm 0.5$ years (range 1-11 years). A total of 30 punch biopsies were performed, in 19 women (15\%). In 7 women more than 3 biopsies were performed along the time. Half of the biopsies were performed during the first year of follow-up, mostly during the first visit (suspicious lesion, uncertain clinical diagnosis) or non-response to first line treatment. The remaining were performed whenever a suspicious lesion appeared during the follow-up. We had 3 cases $(2.4 \%)$ of vulvar HSIL, with two $(1.6 \%)$ having a subsequent diagnosis of VSCC. No cases of differentiated vulvar intraepithelial neoplasia were observed.

Concerning the 3 cases of HSIL diagnosed, it is relevant to mention that none of those women were smokers, had any type of auto-immune disease or were under systemic immunosuppressive therapy. In case 1, after excisional treatment, the patient was observed at regular 4 months intervals during the first year and then annually; treatment with topical corticosteroid recommend in SOS, with a good compliance to both treatment and follow-up. In case 2, the patient was referred after the diagnosis of VSCC for oncologic treatment consisting of radical vulvectomy with bilateral inguino-femoral lymphadenectomy, and subsequent follow-up every 3 months during the first two years and then every 6 months. In the third case, despite follow-up appointments every 3-4 months, she was diagnosed with VSCC 1,5 years after the diagnosis of HSIL. No evidence of recurrence 5 years after oncologic treatment (surgical treatment plus vulvar radiotherapy).

Table 2 summarizes the data of the cases in which biopsy revealed a pre-malignant/malignant lesion.

Table 2. Data about cases in which the biopsy revealed a pre-malignant/malignant lesion.

\section{Discussion}

To our knowledge, this study is one of the largest series of vulvar LP. The percentage of LP that we found in our clinic is slightly higher than that described in similar series, reinforcing that this vulvar dermatosis is not as rare as previously thought. ${ }^{13}$ To put in perspective, the rate of lichen sclerosus in our clinic, a more frequent and well-known dermatosis, is $21.9 \%$.

Our data confirms previous findings in terms of patients' characteristics and clinical presentation. ${ }^{14}$ Regarding therapeutic decisions, a quarter of our patients needed a second line treatment for disease control and $6.3 \%$ 
needed more than two therapeutic options before experiencing an improvement in their symptoms. The schemes of ultra-potent topical corticosteroid used are similar to the ones described in the literature. ${ }^{15}$

\section{Erosive LP and malignancy risk}

The relation between genital LP and cancer is not well-stablished. There are some reasons pointed to refute an association between vulvar LP and VSCC. First, erosive LP is usually seen on nonkeratinized epithelium of the vestibule and vagina, where primary HPV-independent cancer has seldom been reported. However, establishing the exact location of the malignant and premalignant lesions in relation to the mucocutaneous junction can be difficult, given the anatomical and structural changes often present in the field of vulvar dermatosis or in the presence of locally advanced cancer. Second, LS has consensually been associated with premalignant lesions (d-VIN) in contrast to carcinogenic potential of vulvar LP; the possibility of coexistence of LP and LS in the same patient, can be a confounder factor when analyzing the malignant potential of vulvar LP. ${ }^{2}$ Nevertheless, the existence of case reports and series describing neoplasia in a field of vulvar LP and the association between oral LP and cancer, justifies the need for more evidence. ${ }^{7,8,4,16}$

While we could not establish an association between LP and d-VIN or non-HPV VSCC, we had 3 cases $(2.4 \%)$ of vulvar HSIL, with two $(1.6 \%)$ later having a diagnosis of VSCC. Our rate of malignancy was similar to that found by others: $2.3 \%$ in a total of 175 cases reported in a multicentric vulvar LP case audit in the UK ${ }^{15} ; 2.1 \%$ in 95 cases of LP from a vulvar clinic in Rotterdam ${ }^{17} ; 1.2 \%$ of vulvar HPV-induced HSIL from a total of 584 patients with vulvar $\mathrm{LP}^{4}$; and $0.14 \%$ of vulvar cancer in the largest cohort described of any type and location of $\mathrm{LP}^{7}$. While we cannot exclude an association, the risk, if any, is low. In all the 3 cases we report, a close follow-up was maintained, and the patients were compliant with both treatment and follow-up appointments. Even so, in the case number 2, the short interval between a diagnosis of HSIL and that of VSCC, may be explained by: a) invasion already present, but missed in the biopsy; or b) multicentric lesions with rapid progression.

We cannot determine what was the role of treatment and surveillance, given the low figures. However, the third case is an example of success, highlighting the importance of maintaining close surveillance and performing as many biopsies as necessary when facing a suspicious lesion.

\section{Carcinogenesis in vulvar LP}

No cases of d-VIN were identified in our series. Usually, the malignancy precursor associated to vulvar dermatosis is d-VIN, a more rapidly progressing precursor and not related to HPV. ${ }^{4}$ However, concordant with our findings are the ones published by Regauer S, et al and Preti $\mathrm{M}$, et al that reported cases of LP which developed HPV-dependent vulvar SIL lesions. ${ }^{4,5}$ They both present the theory of immunosuppression caused either by the topical treatments frequently used for this disease and the immunosuppression status that many of these patients have because of the association with autoimmune diseases, as the most probable cause for HPV-related lesions. This immunosuppression can cause a reactivation of a previous undetectable HPV or facilitate the acquisition of a new HPV infection. ${ }^{4}$ Also, the fact that recurrent erosions are a common feature of erosive vulvar LP, may be associated to the increase risk of HPV infection acquisition.

\section{Therapeutic options and malignancy risk}

Embracing the theory of immunosuppression as a player in the carcinogenesis of vulvar LP, the therapeutic choices for this disease can and should be questioned. Some second line drugs such as calcineurin inhibitors (i.e. tacrolimus and pimecrolimus), which are topical alternatives to corticosteroids in the treatment of vulvar LP, have directly been associated with reactivation of HPV in response to local immunosuppression. ${ }^{18}$ Its use has also been associated to VSCC, with 2 cases described in vulvar LS and one in penile LS where a correlation between application site and malignancy occurrence was assumed. ${ }^{19,20}$

Aside from these concerns, it is acknowledged that corticosteroids have an anti-inflammatory effect that reduces local disease activity and the transformation risk associated, via an HPV-independent pathway. ${ }^{5}$ Also, their use has recently been identified as an important resource to reduce the recurrence rate of VSCC and dVIN in patients with LS. ${ }^{21}$ For this reason, the hypothesis that relates immunosuppression induced 
by corticosteroids with the risk of HPV related premalignant and malignant lesions, should not sustain an undertreatment policy for vulvar LP, but other drugs like calcineurin inhibitors should only be used as second line therapy and for short periods. ${ }^{19}$ Another important point in favor of its use is that the risk of HPV-dependent lesions in the territory of vulvar LP seems low.

We believe that use of topical and systemic corticosteroids and other immune modulators should still be the gold standard for these patients. The long-term benefits, both in terms of symptoms control and in reducing the risk of HPV-negative malignancies should support therapeutic decisions. However, the treatment of vulvar LP after a diagnosis of HSIL is challenging and one should consider reducing the dose and frequency of topical corticosteroid used for disease control and choose second line treatments other than calcineurin inhibitors (i.e. retinoids) when necessary.

\section{Strengths and limitations}

The main strength of this study is the total number of vulvar LP cases, gathering both clinical and followup information over a large period. Also, all the cases were diagnosed and treated by a vulvar pathology specialist, diminishing the practice differences between clinicians and some other potential selection bias.

The diagnosis of vulvar LP was made either based on clinical features and/or histology. In most cases, the diagnosis was clinical; histology was reserved to the cases refractory to first line treatments and/or when suspicious lesions were observed. Nevertheless, while some authors defend that clinical appearance are nonspecific for vulvar LP and often shared with other dermatosis, like LS, ${ }^{14,22}$ among different guidelines the use of histology for the diagnosis of vulvar dermatosis is not consensual. ${ }^{23-25}$ In the light of these conflicting positions about the diagnostic criteria of vulvar LP and considering that we normally used the clinical criteria for establishing the diagnosis, it could be seen as a limitation of this study. We cannot exclude that in some of the described cases there was an overlapping of different vulvar dermatosis, such as LP and LS. Future studies about vulvar LP that could use a combination of standard clinicopathologic criteria, like the ones proposed by Day, et al. , could be source of more robust evidence. ${ }^{14}$

Another relevant pitfall in our results is the absence of immunohistochemistry stain for p16 in the HSIL histology. A block-positive p16 is a surrogate marker of transforming infection with HPV high-risk-genotypes, and it allows a more reliable diagnosis of HSIL, when considering differential diagnosis, such as d-VIN or erosive LP. ${ }^{2,14}$

\section{Conclusion}

Our data showed that pre-malignant/malignant transformation in women with vulvar LP under surveillance and adequately treated is low. Maintaining a close follow-up of all patients with LP is the best way of preventing future malignancy and obtain the best disease control. Biopsies should be performed whenever a suspicious lesion appears, anytime during the follow-up, being this the undoubtful role of vulvar biopsies.

Competing interest statement: All authors have completed the Unified Competing Interest form and declare: no support from any organisation for the submitted work; no financial relationships with any organisations that might have an interest in the submitted work in the previous three years, no other relationships or activities that could appear to have influenced the submitted work.

\section{Transparence Statement}

The lead author affirms that this manuscript is an honest, accurate, and transparent account of the study being reported; that no important aspects of the study have been omitted; and that any discrepancies from the study as planned (and, if relevant, registered) have been explained.

Contributors: JL and PVB contributed equally to this article. JL and PVB conceptualized the paper. JL analysed the data, with input from CM, RF, RPV and VF. JL and CM wrote the initial draft with all authors providing critical feedback and edits to subsequent revisions. All authors approved the final draft of the manuscript. PVB is the guarantor. The corresponding author attests that all listed authors meet authorship criteria and that no others meeting the criteria have been omitted. 
Ethics approval: This study received the Ethics Committee of Centro Hospitalar Universitário São João approval, $\mathrm{n}^{\mathrm{O}} 182 / 19$.

Funding : no funding

1. Pérez-Lópes F. Postmenopausal Diseases and Disorders. Postmenopausal Diseases and Disorders. 2019.

2. Day T, Otton G, Jaaback K, Weigner J, Scurry J. Is Vulvovaginal Lichen Planus Associated with Squamous Cell Carcinoma? J Low Genit Tract Dis. 2018;22(2):159-65.

3. Day T, Bowden N, Jaaback K, Otton G, Scurry J. Distinguishing erosive lichen planus from differentiated vulvar intraepithelial neoplasia. J Low Genit Tract Dis. 2016;20(2):174-9.

4. Regauer S, Eberz B, Reich O. Human Papillomavirus-Induced Squamous Intraepithelial Lesions in Vulvar Lichen Planus. J Low Genit Tract Dis. 2016;20(4):360-4.

5. Preti M, Micheletti L, Privitera S, Radici G, Gallio N, Benedetto C BL. Vulvar Lichen Planus: A Risk Factor for Vulvar High-Grade Squamous Intraepithelial Lesion Recurrence?

6. Preti M, Rotondo JC, Holzinger D, Micheletti L, Gallio N, McKay-Chopin S, et al. Role of human papillomavirus infection in the etiology of vulvar cancer in Italian women. Infect Agent Cancer. 2020;15(1):411.

7. Halonen P, Jakobsson M, Heikinheimo O, Riska A, Gissler M, Pukkala E. Cancer risk of Lichen planus: A cohort study of 13,100 women in Finland. Int J Cancer. 2018;142(1):18-22.

8. Regauer S, Reich O, Eberz B. Vulvar cancers in women with vulvar lichen planus: A clinicopathological study. J Am Acad Dermatol. 2014;71(4):698-707.

9. Lynch PJ. 2006 International Society for the Study of Vulvovaginal Disease classification of vulvar dermatoses: a synopsis. Vol. 11, Journal of lower genital tract disease. United States; 2007. p. 1-2.

10. Lynch PJ, Moyal-barrocco M, Bogliatto F, Micheletti L, Scurry J. 2006 ISSVD Classification of Vulvar Dermatoses. J Reprod Med. 2006;3-9.

11. Lynch PJ, Moyal-Barracco M, Scurry J, Stockdale C. 2011 ISSVD terminology and classification of vulvar dermatological disorders: An approach to clinical diagnosis. J Low Genit Tract Dis. 2012;16(4):339-44.

12. Simpson RC, Thomas KS, Leighton P, Murphy R. Diagnostic criteria for erosive lichen planus affecting the vulva: An international electronic-Delphi consensus exercise. Br J Dermatol. 2013;169(2):337-43.

13. Micheletti L Bogliatto F, Zanotto-Valentino MC, Ghiringhello B, Massobrio M PM. Vulval lichen planus in the practice of a vulval clinic. Br J Dermatol 2000;143(6)1349-50. 2000;

14. Day T, Wilkinson E, Rowan D, Scurry J. Clinicopathologic Diagnostic Criteria for Vulvar Lichen Planus. J Low Genit Tract Dis. 2020;00(00):1.

15. Simpson RC, Littlewood SM, Cooper SM, Cruickshank ME, Green CM, Derrick E, et al. Real-life experience of managing vulval erosive lichen planus: A case-based review and U.K. multicentre case note audit. Br J Dermatol. 2012;167(1):85-91.

16. Mb CMRF, Torgerson RR, Davis MDP. patients at Mayo Clinic. J Am Dermatology [Internet]. 2017;77(6):1053-9. Available from: https://doi.org/10.1016/j.jaad.2017.07.030

17. Santegoets LAM, Helmerhorst TJM, Van Der Meijden WI. A retrospective study of 95 women with a clinical diagnosis of genital lichen planus. J Low Genit Tract Dis. 2010;14(4):323-8.

18. Bilenchi R, Poggiali S, De Padova LA, Pisani C, De Paola M, Fimiani M. Human papillomavirus reactivation following topical tacrolimus therapy of anogenital lichen sclerosus [21]. Br J Dermatol. 2007;156(2):405-6. 
19. Edey K, Bisson D, Kennedy C. Topical tacrolimus in the management of lichen sclerosus [4]. BJOG An Int J Obstet Gynaecol. 2006;113(12):1482.

20. Ormerod AD. Topical tacrolimus and pimecrolimus and the risk of cancer: How much cause for concern? Br J Dermatol. 2005;153(4):701-5.

21. Chin S, Scurry J, Bradford J, Lee G, Fischer G. Association of Topical CorticosteroidsWith Reduced Vulvar Squamous Cell Carcinoma Recurrence in PatientsWith Vulvar Lichen Sclerosus. JAMA Dermatol.

22. Howard M, Hall A. Vulval lichen planus-lichen sclerosus overlap. Int J STD AIDS. 2018;29(10):1017-23.

23. ACOG. Clinical Management Guidelines for Obstetrician - Gynecologists. Obstet Gynecol. 2019;133(76):168-86.

24. van der Meijden WI, Boffa MJ, ter Harmsel WA, Kirtschig G, Lewis FM, Moyal-Barracco M, et al. 2016 European guideline for the management of vulval conditions. J Eur Acad Dermatology Venereol. 2017;31(6):925-41.

25. Edwards SK, Bates CM, Lewis F, Sethi G, Grover D. 2014 UK national guideline on the management of vulval conditions. Int J STD AIDS. 2015;26(9):611-24.

\section{Hosted file}

Table_BJOG.docx available at https://authorea.com/users/326778/articles/454550-erosivevulvar-lichen-planus-and-risk-of-vulvar-neoplasia-retrospective-case-series-review 